\title{
Qualidade de vida entre obesos mórbidos e pacientes submetidos à cirurgia bariátrica
}

\section{Quality of life among morbid obese and patients submitted to bariatric surgery}

\author{
Lívia Moreira Barros ${ }^{1}$, Rosa Aparecida Nogueira Moreira ${ }^{2}$, \\ Natasha Marques Frota ${ }^{3}$, Thiago Moura de Araújo ${ }^{4}$, Joselany Áfio Caetano ${ }^{5}$ \\ ${ }^{1}$ Enfermeira, Mestre em Enfermagem. Discente do Programa de Pós-Graduação em Enfermagem, nível Doutorado, da Universidade Federal do Ceará (UFC). Fortaleza, \\ CE, Brasil. E-mail: livia.moreirab@hotmail.com. \\ ${ }^{2}$ Enfermeira, Mestre em Enfermagem. Enfermeira da Unidade de Pediatria do Hospital Municipal de Maracanaú Dr. Elívio Holanda. Fortaleza, CE, Brasil. E-mail: \\ nogueiramoreira@bol.com.br. \\ ${ }^{3}$ Enfermeira, Mestre em Enfermagem. Discente do Programa de Pós-Graduação em Enfermagem, nível Doutorado, da UFC. Fortaleza, CE, Brasil. E-mail: \\ enfanatashafrota@yahoo.com.br. \\ ${ }^{4}$ Enfermeiro, Doutor em Enfermagem. Professor Adjunto da Universidade da Integração Internacional da Lusofonia Afro-Brasileira. Redenção, CE, Brasil. E-mail: \\ thiagomouraenf@yahoo.com.br. \\ ${ }^{5}$ Enfermeira, Doutora em Enfermagem. Professor Adjunto da Faculdade de Farmácia, Odontologia e Enfermagem da UFC. Fortaleza, CE, Brasil. E-mail: joselany@ufc.br.
}

\section{RESUMO}

Objetivou-se avaliar a Qualidade de Vida (QV) entre os obesos que aguardam a realização da cirurgia bariátrica e pacientes que estão no pós-cirúrgico. Estudo transversal com abordagem quantitativa realizado em um hospital referência em cirurgia bariátrica em Fortaleza-CE. A amostra foi de 64 pacientes do pré-operatório e 92 do pósoperatório. Dados coletados por meio do questionário de QV de Moorehead-Ardelt II do protocolo Bariatric Analisys and Reporting Outcome System (BAROS). A maioria dos indivíduos do período do pré-operatório classificou sua QV como mínima - 57,8\% (37), enquanto que, no grupo do pós-operatório, observou-se uma mudança significativa em que 75\% (69) dos participantes consideraram que sua QV estava muito boa após a cirurgia $(p<0,001)$. Em ambos os grupos, os aspectos da QV que apresentaram pior avaliação foram: atividade física, interesse sexual e comportamento alimentar. A cirurgia bariátrica representa uma melhora na qualidade de vida dos indivíduos.

Descritores: Obesidade Mórbida; Cirurgia Bariátrica; Qualidade de Vida; Perda de Peso; Enfermagem Perioperatória.

\section{ABSTRACT}

The objective was to assess Quality of Life (QoL) among obese waiting for bariatric surgery and post-surgery patients. A cross-sectional quantitative study conducted in a hospital reference in bariatric surgery in Fortaleza-CE. The sample was composed by 64 pre- and 92 post-surgery patients. Data was collected through the QoL questionnaire MooreheadArdelt II from the Bariatric Analysis and Reporting Outcome System (BAROS) protocol. Most individuals at pre-surgery stage classified their QoL as minimal - 57,8\% (37), while the post-surgery group observed a significant change where $75 \%$ (69) of participants considered their QoL very good after the procedure $(p<0,001)$. In both groups, the QoL aspects that presented worse evaluation were: physical activity, sexual interest and eating behavior. Bariatric surgery represents improvement in quality of life of individuals.

Descriptors: Obesity, Morbid; Bariatric Surgery; Quality of Life; Weight Loss; Perioperative Nursing. 


\section{INTRODUÇÃO}

Nas últimas décadas, observa-se um aumento contínuo no número de pessoas obesas ou com excesso de peso, o que tornou a obesidade um dos principais problemas de saúde no mundo contemporâneo ${ }^{(1-3)}$. A Qualidade de Vida (QV) desses indivíduos obesos está afetada quando comparada com a de pessoas com peso adequado e o crescimento nas taxas de obesidade equivale, portanto, a um aumento contínuo no grupo de pessoas com menor qualidade de vida ${ }^{(1,4)}$.

Um método eficaz para o tratamento de pacientes com obesidade grave é a cirurgia bariátrica, a qual resulta em uma perda de peso significativa e melhoria das comorbidades e da qualidade de vida ${ }^{(1,5-6)}$. A expectativa de melhoria na QV representa um dos principais motivos para pacientes que procuram o controle da obesidade por intermédio da cirurgia bariátrica ${ }^{(1,4-5,7)}$.

Entre os obesos mórbidos, há a expectativa de que a redução do peso e o desaparecimento ou a diminuição dos sintomas associados à obesidade após a cirurgia bariátrica poderão resultar em melhora da qualidade de vida, manifestada no desempenho do trabalho, no relacionamento social e na rotina diária, como, por exemplo, vestir uma roupa ou ser visualizado por outrem $^{(8)}$.

Os resultados das cirurgias bariátricas não dependem exclusivamente da perda de peso, mas também da resolução de comorbidades e melhoria da qualidade de vida $^{(4,6-7)}$. Assim, a avaliação da qualidade de vida relacionada ao estado de saúde é um fator importante na verificação do impacto causado pela doença no bem estar do indivíduo, em especial naqueles que apresentam outras comorbidades. Dessa forma, para que a avaliação dos resultados da cirurgia bariátrica nos domínios que envolvem a qualidade de vida seja feita de forma eficaz, é necessário dispor de instrumentos confiáveis, pois essa mensuração permite a obtenção de dados sobre a eficácia do procedimento cirúrgico na vida diária desses indivíduos no pós-operatório ${ }^{(1,9-10)}$.
A importância do estudo da qualidade de vida no processo terapêutico da cirurgia bariátrica é confirmado por numerosos estudos avaliando o efeito da obesidade no estado de bem-estar e de satisfação com a vida em pacientes no pós-operatório ${ }^{(1-2,6)}$. Com o objetivo de mensurar a QV, diversos instrumentos foram desenvolvidos nos últimos anos e, para a cirurgia bariátrica, destaca-se o questionário de avaliação da qualidade de vida específico para essa população, o qual está incluído no Protocolo BAROS (Bariatric Analysis and Reporting Outcome System), elaborado por Oria e Moorehead, que avalia os resultados do tratamento cirúrgico $^{(1)}$. É uma ferramenta padronizada projetada especificamente para avaliar os resultados psicossociais existentes após a cirurgia bariátrica e é aplicável nos pacientes antes ou depois da cirurgia bariátrica ${ }^{(9)}$.

Embora seja amplamente demonstrado que a cirurgia bariátrica oferece, para muitos pacientes, a melhora a curto e a longo prazo na qualidade de vida física, psicológica, social e sexual, podem haver variações nas alterações pós-operatórias em cada paciente e em cada uma dessas dimensões da $Q V^{(4-6)}$. Assim, após a cirurgia, é necessário a observação a longo prazo da qualidade de vida com o intuito de verificar quais aspectos estão apresentando melhorias, devendo 0 enfermeiro estabelecer estratégias que possibilitem o alcance do bem-estar no decorrer do pós-operatório.

A avaliação de procedimentos de grande porte que irão proporcionar mudanças permanentes na vida do indivíduo deve ser feita periodicamente no pósoperatório com o intuito de verificar a adaptação do paciente ao novo estilo de vida. O questionário de qualidade de vida Moorehead-Ardelt II realiza a avaliação com efetividade, pois permite identificar as alterações percebidas pelos pacientes na sua qualidade de vida após a cirurgia. Pode ser aplicado com pacientes do pré e do pós-operatório para se comparar as mudanças ocorridas na qualidade de vida em benefício da realização da cirurgia bariátrica. 
O uso de instrumentos padronizados favorece 0 planejamento das ações no cuidado ao cliente com a implementação das intervenções e a avaliação do resultado das mesmas. Além disso, é importante que os profissionais ampliem a sua participação nos cuidados direcionados ao paciente bariátrico durante todo o seu percurso em busca do peso desejado, sendo a avaliação da qualidade de vida e a orientação sobre como obter um estilo de vida mais saudável uma das formas de prestar o cuidado. Além disso, a avaliação da qualidade de vida pode proporcionar o conhecimento dos fatores que devem ser alvo das intervenções de enfermagem visando a melhoria da qualidade de vida após a cirurgia e a prestação de uma assistência ao paciente mais eficaz.

Diante do exposto, esse estudo teve como objetivo avaliar a qualidade de vida entre os obesos que aguardam a realização da cirurgia bariátrica e pacientes que estão no pós-cirúrgico.

\section{MÉTODOS}

Estudo transversal realizado no período de novembro de 2011 a junho de 2012 em um hospital referência na realização de cirurgias bariátricas pelo Sistema Único de Saúde (SUS) no município de Fortaleza-Ceará. Essa instituição realiza a cirurgia bariátrica desde 2002 com realização mensal, em média, de oito cirurgias. O hospital também proporciona o acompanhamento do paciente pela equipe multiprofissional antes da cirurgia e até cinco anos após o procedimento.

A população-alvo foi composta pelos pacientes do Programa de Obesidade do Estado do Ceará, que de acordo com dados do Núcleo de Epidemiologia da instituição, estima-se que, em outubro de 2011, 300 pacientes do pré-operatório estavam no processo de realização de consultas com a equipe multiprofissional e exames para tornarem-se aptos a realizarem a cirurgia e 563 pacientes já estavam vivenciando o pós-operatório da cirurgia bariátrica. Dessa forma, a amostra do estudo foi por temporalidade, sendo dividida em dois grupos: pacientes do pré-operatório e pacientes do pósoperatório.

A amostra do pré-operatório foi composta por 64 pacientes que estão na lista de espera para a realização da cirurgia bariátrica. Os critérios de inclusão do grupo do pré-operatório foram: a) idade superior a 18 anos; b) estar no pré-operatório da cirurgia bariátrica; c) estar fazendo o acompanhamento com a equipe multidisciplinar e d) ter comparecido para atendimento na instituição no período da coleta de dados. Como critérios de exclusão temos: pacientes que possuíam alguma limitação cognitiva de modo a comprometer a resposta ao instrumento de coleta de dados tendo em vista que o questionário é autoaplicável.

Já a amostra do pós-operatório foi composta por 92 pacientes bariátricos que estavam no pós-operatório tardio e compareceram ao ambulatório da instituição no período referido para a realização de consultas com a equipe multidisciplinar. Os critérios de inclusão foram: a) idade superior a 18 anos; b) estar vivenciando o pósoperatório há, no mínimo, três meses. O critério de exclusão foi paciente que possuía alguma limitação cognitiva de modo a comprometer a participação na coleta de dados.

Para o grupo do pós-operatório, foi estabelecido o tempo mínimo de três meses devido à recomendação presente na portaria de Assistência de Alta Complexidade ao Paciente Portador de Obesidade Grave e as Diretrizes para a Atenção ao Paciente com Obesidade Grave do Ministério que indica que a avaliação da qualidade de vida, a partir desse instrumento adaptado do BAROS, deve ser feita no $3^{\circ}$ mês do pós-operatório, podendo ser realizada uma avaliação da qualidade de vida ainda no pré-operatório para servir de parâmetro na evolução desse aspecto no pós-operatório ${ }^{(11)}$.

O BAROS é um protocolo que apresenta três principais domínios: avaliação da perda de peso (com a utilização do percentual de redução do excesso de peso), avaliação clínica (através da identificação da melhora ou resolução das comorbidades como doença cardíaca, 
Hipertensão Arterial Sistêmica, Diabetes Mellitus, osteoporose, infertilidade e apneia do sono) e avaliação da qualidade de vida (com o uso do questionário de Moorehead-Ardelt II). Para cada domínio, poderão ser concedidos no máximo três pontos. A ocorrência de complicações e de reoperações diminui os pontos totais. O questionário sobre qualidade de vida é composto por seis questões referentes à auto-estima, à disposição para atividades físicas, ao desempenho no trabalho, à interação social, à prática de atividade sexual e ao comportamento alimentar ${ }^{(12)}$.

Os dados foram coletados diretamente com o cliente na consulta ambulatorial a partir de uma entrevista com o preenchimento de um instrumento estruturado que continha informações sobre os dados sociodemográficos (sexo, idade, estado civil, religião, ocupação, renda familiar e procedência) e o questionário de Qualidade de Vida elaborado por Moorehead-Ardelt, o qual é disponibilizado na Portaria no 492 de 31 de agosto de 2007 do Ministério da Saúde. O questionário é autoaplicável e contém seis itens que avaliam subjetivamente a qualidade de vida do paciente: 1) autoestima, 2) atividade física, 3) relações sociais, 4) satisfação no trabalho, 5) prazer relacionado à sexualidade e 6) o comportamento alimentar. Todas as questões têm o mesmo peso e uma escala de Likert de 1 a 10 é utilizada para marcar as respostas que variam de 0,5 a 0,5. Cada questão vale 0,5 pontos, somando um total de três pontos para o domínio de qualidade de vida. Após a totalização dos escores, a qualidade de vida é classificada como muito ruim $(-3$ a $-2,1)$, ruim $(-2$ a $-1,1)$, mínima (-1 a 1$)$, boa $(1,1$ a 2$)$ e muito boa $(2,1 \text { a } 3)^{(13)}$.

Para a análise dos dados, as informações foram tabuladas no Excel e exportadas para o software estatístico SPSS, versão 19, para tratamento e geração dos resultados. Os dados relativos às variáveis sociodemográficas dos pacientes foram tratados a partir das frequências absolutas e relativas. Para avaliar a normalidade das médias das pontuações entre os grupos do pré e do pós-operatório, utilizou-se o teste de
Kolmogorov-Smirnov e Shapiro-Wilk. Para comparar a melhora da qualidade de vida com a classificação da pontuação do protocolo BAROS de cada paciente do pósoperatório, foi utilizado de Teste de Mann-Whitney. O nível de significância adotado foi de $5 \%$ e o intervalo de confiança de $95 \%$.

O estudo obedeceu às recomendações da Resolução 466/2012 do Conselho Nacional de Saúde que regulamenta a pesquisa com seres humanos no Brasil. $O$ projeto foi aprovado pelo Comitê de Ética em Pesquisa da instituição (CEP538/2011). Antes de iniciar a coleta, os indivíduos foram novamente esclarecidos sobre os objetivos da pesquisa e sigilo dos dados, sendo solicitada a assinatura do Termo de Consentimento Livre e Esclarecido (TCLE).

\section{RESULTADOS}

O perfil do grupo estudado aponta o predomínio do sexo feminino em ambos os grupos em que a frequência de mulheres foi de $87,5 \%$ (56) no pré-operatório e $82,6 \%$ (76) no pós-operatório $(p=0,501)$. Com relação à idade, também houve semelhança na faixa etária dos participantes, pois o maior percentual encontrava-se na faixa etária de 29 a 38 anos, sendo a frequência de 42,2\% no pré e $33,7 \%$ no pós-operatório $(p=0,109)$. A média de idade foi de $35,47 \pm 9,51$ anos no grupo do pré-operatório e uma variância que abrange de 20 a 57 anos. Já no pósoperatório, a média de idade é de 40,53 \pm 10,03 anos no grupo do pré-operatório e uma variância que abrange de 22 a 70 anos $(p=0,003)$.

Quanto ao estado civil, 50\% (32) dos indivíduos do pré-operatório eram casados. Já no grupo do pósoperatório, o matrimônio esteve presente em 65,2\% (60) dos pacientes, havendo também semelhança entre os grupos nessa característica sociodemográfica $(p=0,149)$. Em relação ao grau de escolaridade, houve o predomínio do ensino médio completo em ambos os grupos em que 46,9\% (30) dos pacientes do pré-operatório e 53,3\% (49) do pós-operatório haviam concluído o ensino médio $(p=0,368)$. 
Ao se avaliar a ocupação dos indivíduos de ambos os grupos, observou-se que $68,7 \%$ (44) dos pacientes do pré e $75 \%$ (69) do pós-operatório exerciam atividade remunerada. A distribuição da renda familiar mensal foi semelhante nos dois grupos visto que a maioria apresentou renda familiar entre um a três salários mínimos (R\$ 622,00 por salário), sendo a frequência de $51,6 \%$ (33) no pré-operatório e 40,2\% (37) no pósoperatório $(p=0,089)$.

Quanto à procedência, observa-se que ainda uma maior participação no programa de obesidade de indivíduos que residem na Região Metropolitana de Fortaleza, pois $65,6 \%$ (42) dos pacientes do pré e 75\% (69) do pós-operatório procediam de Fortaleza e sua Região Metropolitana. Apenas 32,8\% (21) e 21,7\% (20) dos pacientes do pré e do pós-operatório, respectivamente, procediam da zona rural $(p=0,266)$.

Apresenta-se, no Gráfico 1, a classificação dos pacientes dos grupos do pré e do pós-operatório sobre a qualidade de vida.

\section{Qualidade de Vida}

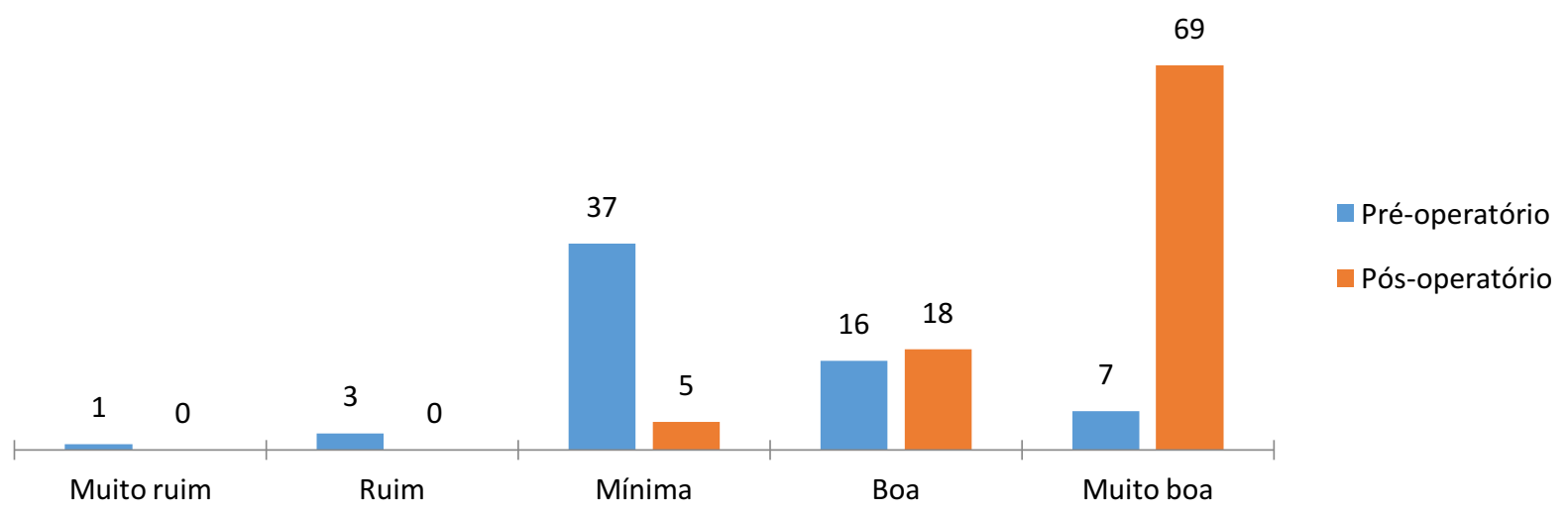

Gráfico 1: Distribuição da classificação da qualidade de vida entre os grupos do pré e do pós-operatório dos pacientes do Programa de Obesidade do Estado do Ceará (n=156). Fortaleza, CE, Brasil, 2012.

Os dados obtidos por meio do Questionário de Qualidade de Vida de Moorehead-Ardelt II demonstraram que $57,8 \%$ (37) dos indivíduos do período do préoperatório classificaram sua qualidade de vida como mínima e apenas $10,9 \%$ (sete) a consideraram como muito boa e $25 \%$ (16) como boa. No grupo do pósoperatório, observou-se uma mudança significativa no resultado dessa classificação em que 75\% (69) dos participantes consideraram sua qualidade de vida muito boa após a realização da cirurgia, 19,6\% (18) sentem que a QV está boa e apenas cinco $(5,4 \%)$ pacientes classificaram a QV como mínima, não havendo nesse grupo nenhuma classificação da QV como ruim ou muito ruim.
Para identificar as mudanças ocorridas na qualidade de vida entre os grupos do pré e do pós-operatório apresentadas na Tabela 1, apresenta-se abaixo a correlação entre os domínios avaliados na qualidade de vida e a sua significância estatística (Tabela 1). 
Tabela 1: Média (desvio padrão) da pontuação de cada domínio da qualidade de vida entre os grupos do pré e do pós-operatório dos pacientes do Programa de Obesidade do Estado do Ceará ( $n=156)$. Fortaleza, CE, Brasil, 2012.

\begin{tabular}{cccc}
\hline \multirow{2}{*}{ Estatísticas Descritivas } & \multicolumn{2}{c}{ Pontuação da Qualidade de Vida Média (Desvio Padrão) } & \multirow{2}{*}{ p-valor* } \\
\cline { 2 - 3 } & $0,794(1,13)$ & $2,335(0,65)$ & $<0,001$ \\
Pontuação Total & $0,800(0,28)$ & $0,459(0,09)$ & $<0,001$ \\
Auto-estima & $-0,420(0,35)$ & $0,214(0,27)$ & $<0,001$ \\
Atividade física & $0,238(0,33)$ & $0,404(0,18)$ & $<0,001$ \\
Sociabilidade & $0,231(0,28)$ & $0,441(0,14)$ & $<0,001$ \\
Desempenho no trabalho & $0,062(0,37)$ & $0,326(0,27)$ & $<0,001$ \\
Interesse sexual & $0,144(0,32)$ & $0,395(0,14)$ & $<0,001$ \\
Comportamento alimentar & & &
\end{tabular}

Com relação aos seis domínios abordados no questionário, todos apresentaram comparações estatísticamente significantes entre os dois grupos com $\mathrm{p}<0,001$ para todos os domínios. No período do préoperatório, os domínios que tiveram a menor média foram: a atividade física, o interesse sexual e o comportamento alimentar. Foi observada uma melhora na avaliação da qualidade de vida nesses domínios entre os pacientes do pós-operatório. Entretanto, esses domínios ainda se apresentaram como os aspectos da QV com menores médias (Tabela 1).

Apesar do domínio referente à atividade física ter tido uma das menores pontuações em ambos os grupos, destaca-se que esse aspecto foi o que teve a maior variação tendo em vista que a média da pontuação variou de -0,420 para 0,214 quando comparamos o grupo do pré e do pós-operatório, respectivamente (Tabela 1).

\section{DISCUSSÃO}

A cirurgia bariátrica melhora a qualidade de vida e a percepção sobre o estado de saúde, sendo essas mudanças observadas no primeiro ano de pósoperatório, podendo durar até 10 anos $^{(14-15)}$. Está associada com redução da depressão e agressividade, melhora do autoconceito, da autoestima, dos sentimentos, da satisfação e da capacidade de realizar atividades. Promove melhoras também no conforto, mobilidade, envolvimento social, funcionamento sexual e na produtividade ${ }^{(13,15-16)}$.
Nesse estudo, houve uma melhora significativa da qualidade de vida das pessoas que se submeteram à cirurgia bariátrica. Outro estudo ao avaliar a QV em pacientes antes e após a cirurgia bariátrica em um sistema público de saúde brasileiro verificou que, após a cirurgia, $82,2 \%$ dos pacientes consideraram sua qualidade de vida como boa ou muito boa, enquanto que, no préoperatório, apenas $40 \%$ consideraram dessa mesma forma a $Q V^{(10)}$.

Além dos problemas de saúde associados à obesidade, observa-se também o prejuízo nos aspectos psicológicos, emocionais ou sociais, os quais são refletidos no déficit da qualidade de vida como intolerância ao exercício físico, dificuldade na mobilidade, dor nas articulações, falta de aceitação de si mesmo, aumento do nível de estresse, diminuição da autoestima e do humor e depressão ${ }^{(1)}$. Os obesos frequentemente relatam menor QV em aspectos como autoestima, relacionamentos amorosos, vida no trabalho e socialização ${ }^{(17)}$.

Os fatores que são particularmente importantes na QV podem fornecer informações sobre o que motiva os pacientes obesos que procuram a cirurgia bariátrica como tratamento. Também permite identificar as metas de resultados na QV mais relevantes para avaliar a eficácia global das intervenções para a perda de a partir da perspectiva dos pacientes ${ }^{(17)}$.

Os piores domínios relacionados à QV são representados pela atividade física, relacionamento 
sexual e comportamento alimentar. Em um estudo realizado com 570 obesos que procuraram a cirurgia bariátrica, os domínios que foram associados com a baixa QV devido à obesidade foram: atividade física, sociabilidade, autoestima e atividade sexual ${ }^{(17)}$.

No presente estudo, a autoestima foi o domínio que obteve a maior pontuação entre ambos os grupos, apesar de termos observado uma diferença negativa na média da pontuação ao compararmos os pacientes do período do pré e do pós-operatório. A autoestima e a autoimagem fazem parte do autoconhecimento. Quando a autoimagem encontra-se em equilíbrio, é possível ter uma análise melhor do seu corpo, o que reflete em uma maior vitalidade, a vaidade e a alegria ${ }^{(18)}$.

Os obesos apresentam baixa autoestima devido à dificuldade na perda de peso, descontentamento com a autoimagem e a aparência, apresentando comportamentos de esquiva como isolamento social. A insatisfação com a imagem corporal é fortemente correlacionada com sintomas de depressão, o que é particularmente verdadeiro em mulheres devido, provavelmente, a ênfase social sobre o físico feminino $^{(15,19)}$.

Com relação ao domínio da atividade física, observou-se uma melhora nas pontuações entre o grupo do pré e do pós-operatório, fato este que pode estar relacionado à contribuição da realização da cirurgia bariátrica para o abandono do sedentarismo e o início da prática da atividade física. Entretanto, apesar da melhora na qualidade de vida após a cirurgia, alguns pacientes ainda demonstram alguns obstáculos em praticar atividade física como falta de interesse, tempo e motivação.

Existe uma associação positiva entre atividade física e perda de peso no pós-operatório. Os pacientes que perdem mais peso pode se sentir melhor fisicamente e isso pode levá-los a se exercitar mais ${ }^{(20)}$. Os benefícios do exercício incluem aumento do gasto de energia e de massa corporal magra, melhora da autoestima, do humor, da depressão e da qualidade de vida, perda de gordura e diminuição do risco de complicações relacionadas com a obesidade $\mathrm{e}^{(20-21)}$.

A prática de atividade física é, muitas vezes, difícil para os pacientes com obesidade mórbida devido à presença de comorbidades, tais como osteoartrite e asma, e à falta de condicionamento físico ${ }^{(20)}$. A obesidade dificulta a deambulação do indivíduo, pois, na tentativa de minimizar o impacto do peso nas articulações do joelho e na ocorrência de dores, o obeso tende a ter uma marcha mais lenta, o que desmotiva a prática de atividades físicas $^{(3)}$.

As rápidas mudanças físicas após a cirurgia tendem a aumentar a QV e motivar o indivíduo a se comprometer com o seu novo estilo de vida a fim de manter o sucesso do tratamento cirúrgico a longo prazo. Logo nos três primeiros meses após a cirurgia, observa-se melhorias na marcha, na velocidade das caminhadas, no condicionamento físico e nas dores nas articulações e na região lombar, o que contribui para uma melhor autopercepção e QV. Essas mudanças podem, ainda, facilitar a participação em atividades físicas regulares, possibilitando uma maior perda de peso ${ }^{(3)}$.

Após a cirurgia, é possível perceber uma maior inserção do indivíduo nas atividades sociais tendo em vista que os pacientes do pós-operatório pontuaram melhor no domínio da sociabilidade, revelando uma satisfação em se envolver em reuniões sociais e familiares, além de estarem mais comunicativos. A cirurgia bariátrica representa, então, um renascimento e uma transformação para melhor ${ }^{(14-15)}$.

O isolamento social associado à obesidade é um dos fatores mais importantes que contribuem para o déficit na qualidade de vida ${ }^{(19)}$. A melhora na socialização está diretamente associada à melhora da QV e as mudanças que ocorrem com a perda de peso como conseguir sentarse num barzinho (sem preocupar-se com a cadeira), ir ao cinema, viajar de avião, andar de ônibus, entrar numa piscina, ir à praia, recuperar o direito de ir e vir, não precisar de ajuda para levantar-se de um sofá ou mesmo para sua higiene pessoal, assumir responsabilidades das 
quais eram poupadas, por falta de condições físicas e pelas limitações que a obesidade impunha ${ }^{(22-23)}$.

A capacidade de trabalhar também teve uma mudança significativa quando comparamos pessoas que não realizaram a cirurgia bariátrica com pacientes já operados. Ao contrário de muitas condições crônicas de saúde, a obesidade prejudica a qualidade de vida não só por causa de predispor a ocorrência de outras doenças, mas também por ser, em muitas culturas, socialmente indesejável e estigmatizante. Pessoas obesas são alvo de preconceito em diversos ambientes como na escola, no trabalho e nos serviços de saúde ${ }^{(17)}$.

Com relação ao interesse sexual, os pacientes do pósoperatório apresentaram uma média maior na pontuação desse domínio. Isso demonstra que a melhora da autoestima pode influenciar também na melhora da vida sexual. Após a cirurgia, ocorrem melhorias significativas no funcionamento sexual, excitação, lubrificação, desejos e satisfação, havendo também melhoria na autoimagem e na satisfação nos relacionamentos amorosos ${ }^{(2)}$.

A sexualidade recebe pontuação positiva na maioria das investigações após a cirurgia bariátrica, mas casos em que há sobra excessiva de pele e falta de condições financeiras para operações consideradas estéticas, levam ao retraimento da pessoa e até ao sentimento de mais uma falência. $\mathrm{O}$ excesso de pele pode causar desconforto, principalmente durante os relacionamentos sexuais, o que pode favorecer o surgimento de situações de vergonha da autoimagem, representando um lembrete constante da impossibilidade de se libertar totalmente do grande corpo obeso $^{(14)}$.

O comportamento alimentar avalia a forma como o paciente aproveita a comida. Um dos objetivos da cirurgia bariátrica é promover uma perda de peso satisfatória e permanente associada a uma melhor qualidade na alimentação. Geralmente, pacientes que ainda não realizaram a cirurgia bariátrica mantém hábitos alimentares inadequados em que esses indivíduos não escolhem alimentos nutritivos e não saboreiam esses alimentos ao deglutirem apressadamente. Essa mudança nos hábitos alimentares pode ser observado nos grupos desse estudo em que houve uma melhora da média da pontuação do domínio do comportamento alimentar.

A cirurgia bariátrica é um procedimento invasivo que afeta o comportamento alimentar, devendo 0 profissional de saúde investigar os fatores de risco potenciais para o fracasso na perda de peso a longo prazo $^{(24)}$. Após a cirurgia, os pacientes passam por um processo de aprendizagem de novos hábitos que são incorporados. Eles passam a mudar o comportamento em relação à comida, aprendendo a adequar o tipo e a quantidade de alimentos que o novo estômago tolera e a tolerar os sinais de satisfação do organismo ${ }^{(15,23)}$.

No pós-operatório, é necessário avaliar as motivações do indivíduo para o consumo dos alimentos indicados, as preferências, a tolerância alimentar, a adesão à dieta e a mastigação. Deve-se enfatizar a importância da ingestão adequada de proteínas após a cirurgia, considerando as deficiências de micronutrientes e as alterações pós-cirúrgicas na composição corporal com a perda de peso ${ }^{(7,25)}$.

Com o intuito de prevenir os sintomas indesejáveis gastrointestinais (náuseas, vômitos ou síndrome de dumping), complicações pós-operatórias (deficiências nutricionais) e recuperação do peso), os pacientes são orientados a alterarem a dieta nutricional e o compotamento alimentar, incluindo o consumo de pequenas porções nos horários estabelecidos e a mastigação adequada ${ }^{(23-24)}$. Devem ser instruídos sobre a importância da ingestão de quantidade adequada de proteínas para evitar a perda de massa magra do corpo desproporcional e evitar a ingestão de doces ou alimentos hipecalóricos para evitar síndrome de dumping. Além disso, deve ser alertado sobre a possibilidade do reganho de peso, caso não siga corretamente as orientações ou não modifique os hábitos alimentares ${ }^{(7,25)}$.

Diante do exposto, destaca-se que o questionário de Qualidade de Vida de Moorehead-Ardelt II é um instrumento útil e de fácil aplicação que se apresenta como uma ferramenta importante para a prática de 
profissionais que trabalham com pacientes que se submetem a cirurgia por permitir avaliar diversos domínios que influenciam na qualidade de vida do indivíduo como a autoestima, atividade física, sociabilidade, desempenho no trabalho, interesse sexual e comportamento alimentar. Todos esses domínios tiveram um aumento significativo na pontuação quando comparamos indivíduos que estavam na fila de espera para a cirurgia bariátrica com indivíduos que já se submeteram ao procedimento cirúrgico.

\section{CONCLUSÃO}

A partir dos resultados obtidos nesse estudo, concluise que a cirurgia bariátrica favorece o aumento da percepção dos indivíduos sobre o seu estado de bemestar e a melhora da qualidade de vida. As mudanças observadas na qualidade de vida foram referentes ao aumento da autoestima com relação direta na melhora da vaidade e da autopercepção; à prática de atividade física, a qual contribui para a perda de peso; ao melhor desempenho no trabalho; à vida social mais ativa devido ao bem-estar vivenciado pelos pacientes; ao aumento do interesse sexual com contribuição para a melhora dos relacionamentos pessoais e ao comportamento alimentar mais saudável.

Os piores domínios avaliados foram semelhantes entre os pacientes do pré e do pós-operatório, os quais foram: atividade física, interesse sexual e comportamento alimentar. Esse achado serve como um alerta para os profissionais de saúde que atuam tanto na atenção primária como nos outros níveis de atenção à saúde para a identificação dos fatores que causam esse déficit e para o estabelecimento de intervenções que visem o alcance de uma melhor qualidade de vida nesses domínios.

Como limitação do estudo, destaca-se o fato de que os pacientes dos grupos do pré e do pós-operatório eram diferentes, porém, isso não compromete a inferência dos resultados apresentados, pois identificou-se um perfil sociodemográfico semelhante entre os sujeitos, o que permite inferir que as mudanças observadas na avaliação da qualidade de vida entre esses dois grupos está relacionada aos benefícios proporcionados pela cirurgia bariátrica.

A realização de outros estudos é recomendada, sobretudo, aqueles que visem acompanhar a evolução da avaliação da qualidade de vida entre o período do préoperatório e, pelo menos, após um ano da realização da cirurgia com a aplicação do questionário em intervalos seriados, o que proporcionaria uma análise mais fidedigna dos resultados da cirurgia bariátrica e do impacto da mesma na qualidade de vida do indivíduo após o procedimento cirúrgico.

mass index and obesity-related comorbidities. Diabetes Metab [Internet]. 2013 [acesso em: 30 jun 2015];39(2):148-54. Disponível em: http://dx.doi.org/10.1016/j.diabet.2012.10.008.

5. Raoof M, Näslund I, Rask E, Karlsson J, Sundbom M, Edholm D, et al. Health-Related Quality-of-Life (HRQoL) on an Average of 12 Years After Gastric Bypass Surgery. Obes Surg [Internet]. 2015 [acesso em: 30 jun 2015];25(7):1119-27. Disponível em:

http://dx.doi.org/10.1007/s11695-014-1513-6.

5. Raoof M, Näslund I, Rask E, Karlsson J, Sundbom M, Edholm M. Health-Related Quality-of-Life (HRQoL) on an Average of 12 Years After Gastric Bypass Surgery. Obes Surg. Article in press. 2015.

6. Brunault P, Frammery J, Couet C, Delbachian I, Bourbao-Tournois C, Objois $\mathrm{M}$, et al. Predictors of changes in physical, psychosocial, sexual quality of life, and comfort with food after obesity surgery: a 12-month follow-up study. Qual Life Res [Internet]. 2015 [acesso em: 30 jun 2015];24(2):493-501. Disponível em: http://dx.doi.org/10.1007/s11136-014-0775-8. 
7. Freeman RA, Overs SE, Zarshenas N, Walton KL, Jorgensen JO. Food tolerance and diet quality following adjustable gastric banding, sleeve gastrectomy and Roux-en-Y gastric bypass. Obes Res Clin Pract [Internet]. 2014 [acesso em: 30 jun 2015];8(2):e183-91. Disponível em: http://dx.doi.org/10.1016/j.orcp.2013.02.002.

8. Araújo AA, Brito AM, Ferreira MNL, Petribú K, Mariano MHA. Modificações da qualidade de vida sexual de obesos submetidos à cirurgia de Fobi-Capella. Rev Col Bras Cir [Internet]. 2009 [acesso em: 30 jun 2015];36(1):42-8. Disponível em: http://dx.doi.org/10.1590/S0100-69912009000100009.

9. Campos GM, Rabl C, Roll GR, Peeva S, Prado K, Smith J, al et. Better weight loss, resolution of diabetes, and quality of life for laparoscopic gastric bypass vs banding: results of a 2-cohort pair-matched study. Arch Surg [Internet]. 2011 [acesso em: 30 jun 2015];146(2):149-55. Disponível em: http://dx.doi.org/10.1001/archsurg.2010.316. 10. Khawali C, Ferraz MB, Zanella MT, Ferreira SRG. Evaluation of quality of life in severely obese patients after bariatric surgery carried out in the public healthcare system. Arq Bras Endocrinol \&amp; Metabol [Internet]. 2012 [acesso em: 30 jun 2015];56(1):33-8. Disponível em: http://dx.doi.org/10.1590/S0004-27302012000100006. 11. Portaria no 492, de 31 de agosto de 2007 (BR) [Internet]. Diário Oficial da União. 05 set 2007 [acesso em: 30 jun 2015]. Disponível em: http://bvsms.saude.gov.br/bvs/saudelegis/sas/2007/prt_0492_31_08_ 2007.html.

12. Oria HE, Moorehead MK. Updated Bariatric Analysis and Reporting Outcome System (BAROS). Surg Obes Relat Dis [Internet]. 2009 [acesso em: 30 jun 2015];5(1):60-6. Disponível em:

http://dx.doi.org/10.1016/j.soard.2008.10.004.

13. Neff KJH, Le Roux CW. Bariatric surgery: A best practice article. J Clin Path [Internet]. 2013 [acesso em: 30 jun 2015]; 66(2):90-8. Disponível em: http://dx.doi.org/10.1136/jclinpath-2012-200798. 14. Warholm C, Øien AM, Råheim M. The ambivalence of losing weight after bariatric surgery. Int J Qual Stud Health Well-being [Internet]. 2014 [acesso em: 30 jun 2015];9:22876. Disponível em:

http://dx.doi.org/10.3402/qhw.v9.22876.

15. Jensen JF, Petersen MH, Larsen TB, Jørgensen DG, Grønbaek HN, Midtgaard J. Young adult women's experiences of body image after bariatric surgery: a descriptive phenomenological study. J Adv Nurs [Internet]. 2014 [acesso em: 30 jun 2015];70(5):1138-49. Disponível em: http://dx.doi.org/10.1111/jan.12275.

16. Costa RC, Yamaguchi N, Santo MA, Riccioppo D, Pinto-Junior PE. Outcomes on quality of life, weight loss, and comorbidities after Rouxen-Y gastric bypass. Arq Gastroenterol [Internet]. 2014 [acesso em: 30 jun 2015];51(3):165-70. Disponível em:

http://dx.doi.org/10.1590/S0004-28032014000300002.

17. Wee CC, Davis RB, Huskey KW, Jones DB, Hamel MB. Quality of life among obese patients seeking weight loss surgery: the importance of obesity-related social stigma and functional status. J Gen Intern Med [Internet]. 2013 [acesso em: 30 jun 2015];28(2):231-8. Disponível em: http://dx.doi.org/10.1007/s11606-012-2188-0.

18. Cavalcanti CL, Gonçalves MCR, Cavalcanti AL, Costa SFG, Asciutti LSR. Programa de intervenção nutricional associado à atividade física: discurso de idosas obesas. Cien Saude Colet [Internet]. 2011 [acesso em: 30 jun 2015];16(5):2383-90. Disponível em:

http://dx.doi.org/10.1590/S1413-81232011000500007.

19. Kubik JF, Gill RS, Laffin M, Karmali S. The Impact of Bariatric Surgery on Psychological Health. J Obes [Internet]. 2013 [acesso em: 30 jun 2015];2013:1-5. Disponível em: http://dx.doi.org/10.1155/2013/837989.

20. Livhits M, Mercado C, Yermilov I, Parikh JA, Dutson E, Mehran A, et al. Exercise following bariatric surgery: systematic review. Obes Surg [Internet]. 2013 [acesso em: 30 jun 2015];20(5):657-65. Disponível em: http://dx.doi.org/10.1007/s11695-010-0096-0.

21. Kalarchian MA, Marcus MD, Courcoulas AP, Cheng Y, Levine MD, Josbeno D. Optimizing long-term weight control after bariatric surgery: a pilot study. Surg Obes Relat Dis [Internet]. 2012 [acesso em: 30 jun 2015];8(6):710-5. Disponível em:

http://dx.doi.org/10.1016/j.soard.2011.04.231.

22. Tariq N, Chand B. Presurgical evaluation and postoperative care for the bariatric patient. Gastrointest Endosc Clin N Am [Internet]. 2011 [acesso em: 30 jun 2015];21(2):229-40. Disponível em:

http://dx.doi.org/10.1016/j.giec.2011.02.010.

23. Knutsen IR, Terragni L, Foss C. Empowerment and bariatric surgery: negotiations of credibility and control. Qual Health Res [Internet]. 2013 [acesso em: 30 jun 2015];23(1):66-77. Disponível em: http://dx.doi.org/10.1177/1049732312465966.

24. Overs S, Freeman R, Zarshenas N, Walton K, Jorgensen J. Food Tolerance and Gastrointestinal Quality of Life Following Three Bariatric Procedures: Adjustable Gastric Banding, Roux-en-Y Gastric Bypass, and Sleeve Gastrectomy. Obes Surg [Internet]. 2012 [acesso em: 30 jun 2015];22(4):536-43. Disponível em: http://dx.doi.org/10.1007/s11695011-0573-0.

25. Mancini MC. Bariatric surgery - An update for the endocrinologist. Arq Bras Endocrinol Metabol [Internet]. 2014 [acesso em: 30 jun 2015];58(9):875-88. Disponível em: http://dx.doi.org/10.1590/0004$\underline{2730000003413 .}$.

Recebido: $17 / 11 / 2013$.

Aceito: 11/03/2014.

Publicado: 30/06/2015. 